International Journal of Modern Physics E

(C) World Scientific Publishing Company

\title{
SKYRME FORCES WITH EXTENDED DENSITY DEPENDENCE
}

\author{
B. COCHET, K. BENNACEUR* J. MEYER \\ IPN Lyon, CNRS-IN2P3/UCB Lyon 1 \\ 43, Bd. du 11 novembre 1918 \\ 69622 Villeurbanne cedex, France \\ P. BONCHE, T. DUGUET ${ }^{\dagger}$ \\ Service de Physique Théorique, CEA Saclay \\ 91191 Gif-sur-Yvette cedex, France
}

Received (received date)

Revised (revised date)

\begin{abstract}
A generalized parameterization of the Skyrme effective force is discussed. Preliminary results are presented for infinite symmetric and asymmetric nuclear matter. In particular, it is shown that an enlarged density dependence based on two terms allows to choose independently the incompressibility and the isoscalar effective mass.
\end{abstract}

\section{Introduction}

It is commonly accepted that it does exist a relation between compressibility, effective mass and the density dependence of a given effective force. Studies with Skyrme forces ${ }^{1}$ have shown that the incompressibility $K_{\infty}$ and the effective mass cannot be chosen independently once the analytical form of the (single) densitydependent term has been chosen. This has led to the $\rho^{1 / 6}$ density dependence in Skyrme forces like SkM* which allows a value of $K_{\infty}$ around $220 \mathrm{MeV}$ close to that extracted from the experimental breathing mode analyses ${ }^{2,3}$ and an effective mass $m^{*}$ around $0.7 m$ simultaneously. A general review of the knowledge about compressibility has been recently summarized by G. Colò et al. ${ }^{4}$.

Recently, the density dependence of phenomenological effective interactions, such as Skyrme or Gogny forces, has been revisited in the context of beyond mean field calculations ${ }^{5,6}$. Indeed, while a dependence of the interaction on the density is well established for calculations at the mean field level ${ }^{7}$, no strongly motivated prescription exists when several mean fields are mixed as in the Generator Coordinate Method (GCM) and the Projected Mean Field Method (PMFM). First, an extension of the Goldstone-Brueckner theory has motivated the GCM and the

*bennaceur@ipnl.in2p3.fr

${ }^{\dagger}$ present address: Physics Division, Argonne National Laboratory, Argonne, IL 60439, USA 
PMFM from a perturbative point of view for the first time ${ }^{5}$. In this extended context, a generalized Brueckner $G$ matrix summing particle-particle ladders has been defined and may be used as a reference from which phenomenological interactions in GCM or PMFM calculations should be approximated. It is possible to simplify this in-medium interaction to extend the validity of the Skyrme force ${ }^{8,9}$ and to identify approximately the density dependence originating from Brueckner correlations in the context of mixed nonorthogonal vacua ${ }^{6}$. Consequently, a single density-dependent term has often been used in phenomenological forces, e.g. Gogny ${ }^{10}$ and Skyrme ${ }^{9}$ effective interactions. On the other hand, the density dependence re-normalizing three-body force effects has been shown to be different from the one taking care of Brueckner correlations when going beyond the mean field approximation ${ }^{6}$. Based on this analysis, it is legitimate to redefine the Skyrme interaction at the mean field level. Including two density-dependent terms, each related to a given physical origin, will allow a proper extrapolation of the interaction to GCM and PMFM calculations.

A first attempt is presented in this article, where we concentrate on the practical advantages of having two different density-dependent terms. Our analysis is focused on $\rho^{1 / 3}$ terms which are connected with a $k_{F}$ expansion of the Brueckner $G$ matrix.

\section{Standard Skyrme effective interactions and beyond}

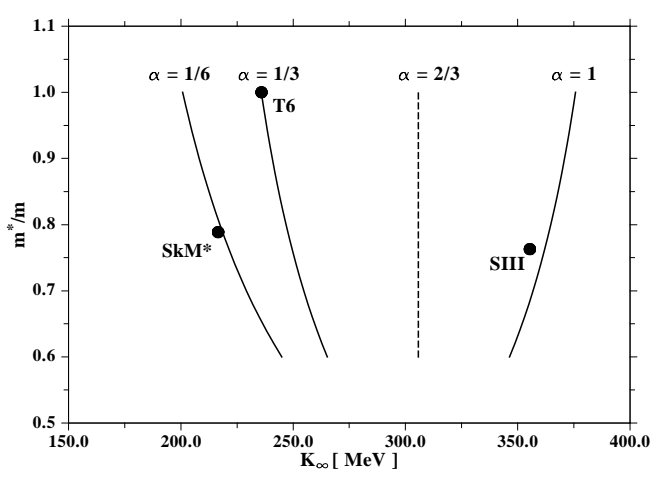

Fig. 1. Correlation between incompressibility, effective mass and density dependence of standard parameterizations of Skyrme effective interactions. When $\alpha=2 / 3$, the value of $K_{\infty}$ is fixed and the effective mass can be freely chosen. However, this case is not interesting since it does not correspond to a realistic value of $K_{\infty}$.

When using Skyrme effective forces with a standard density dependence $\rho^{\alpha}$, where $\rho$ is the total density, the adjustable parameter $\alpha$ is strongly correlated to the incompressibility and the effective mass in nuclear matter. This is illustrated on Figure 1 for different standard Skyrme forces with various powers $\alpha$. In all cases, the different parameters of the force have been adjusted in order to obtain the saturation 
density $\rho_{0}=0.16 \mathrm{fm}^{-3}$ and the energy per particle $E / A=-16 \mathrm{MeV}$ in infinite symmetric nuclear matter. Once these two properties are fixed, the relation between $m^{*} / m$ and $K_{\infty}$ is entirely determined by $\alpha$. This well-known feature, discussed in ref. ${ }^{1}$ yields to the conclusion that a value of $\alpha$ around 1 , like in SIII, does not allow to reach a correct incompressibility ${ }^{2,11}$. Only values of $\alpha$ ranging from $1 / 6$ to $1 / 3$ allow for an acceptable set $\left\{m^{*} / m, K_{\infty}\right\}$.

This constraint disappears with the family of parameterizations presented in Appendix A. Following the previously discussed procedure to adjust the coefficients, but having the additional flexibility coming from the second density dependent term, we can fix the saturation properties of infinite symmetric nuclear matter $\rho_{0}$, $E / A, K_{\infty}$ and $m^{*} / m$ independently and determine the parameters of the force.

However this procedure only guarantees to have realistic properties of nuclear matter in the vicinity of the saturation point. If we want a force which gives an equation of state in agreement with ab initio calculations at low density as well as high density (up to $\sim 3 \rho_{0}$ ) one should adopt a different fitting procedure. First, one can fit a set of points $\left[\rho_{i}, E / A\left(\rho_{i}\right)\right]_{i=1, \ldots, N}$ which samples a realistic equation of state of nuclear matter. In this kind of approach, the presence of a density-dependent term with the power $2 / 3$ in the force is particularly interesting. Indeed, the effective mass is not constrained by the fit and thus, can be freely chosen ${ }^{12}$.

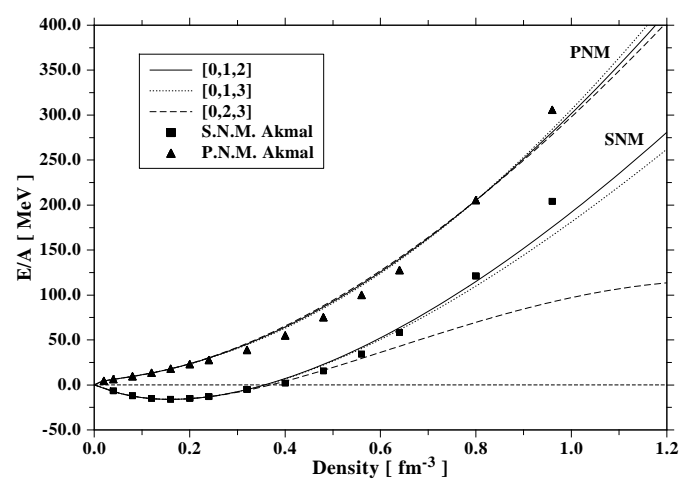

Fig. 2. Energy per particle in infinite matter as a function of equilibrium density. SNM: symmetric nuclear matter; PNM: pure neutron matter. Labels $[0,1,2],[0,1,3],[0,2,3]$ refers to the density dependence of the forces (see text). Full squares and triangles: EOS of Akmal et al. ${ }^{13}$.

The equations of state (EOS) obtained using the generalized forces $[0,1,2]$, $[0,1,3]$ and $[0,2,3]$ (see Appendix A) for symmetric nuclear matter as well as pure neutron matter are presented here. The quantities $\rho_{0}=0.16 \mathrm{fm}^{-3}, E / A=$ $-16 \mathrm{MeV}, K_{\infty}=230 \mathrm{MeV}, m^{*} / m=0.8$ and $\kappa=0.5$ along with a fit of the neutron matter equation of state provided by Akmal et al. ${ }^{13}$ allow the determination of the parameters of the force. Figure 2 shows the two EOS (energy per particle as a function of equilibrium density) for symmetric nuclear matter and pure neutron 
matter compared with the EOS of Akmal et al. ${ }^{13}$. The results are quite reasonable and rather similar for densities from 0 to $\sim 3 \rho_{0}$.

\section{Conclusion}

We have shown that the choice of a standard Skyrme effective force with a modified density dependence based on two terms enables to choose independently the incompressibility and the isoscalar effective mass. The gross properties of nuclear matter investigated here are quite reasonable. With this generalized density dependence we have been able to construct new Skyrme like parameterizations without collapse at relevant densities and which exhibit reasonable equation of state (EOS) for symmetric nuclear matter as well as for pure neutron matter compared to the recent realistic variational EOS of Akmal et al. ${ }^{13}$.

Other choices can be explored for the density dependence provided they are physically well grounded. The choice $\rho^{2 / 3}$ for one of the density-dependent term lets total freedom for the effective mass. This feature is extremely interesting from the perspective of developing accurate and predictive force since it gives a control on the density of state around the Fermi energy where the correlations beyond the Hartree-Fock approximation can develop. Furthermore it implies only two additional parameters so that their total number remains quite small.

\section{Appendix A. Extended parameterizations of Skyrme forces}

The parametrization of the Skyrme forces discussed in this article is

$$
V\left(\mathbf{r}_{1}, \mathbf{r}_{2}\right)=\sum_{i} t_{0 i} \rho^{i / 3}(\mathbf{R})\left(1+x_{0 i} P_{\sigma}\right) \delta(\mathbf{r})+\text { standard non local terms }
$$

In this first exploratory work, we limit our study to the case of density dependent local terms and three non zero values for $t_{0 i}$, namely $i_{1}, i_{2}$ and $i_{3}$ out of the set $\{0,1,2,3\}$. The corresponding forces will be labeled by $\left[i_{1}, i_{2}, i_{3}\right]$.

\section{References}

1. E. Chabanat et al., Nucl. Phys. A627 (1997) 710; ibid. A635 (1998) 231.

2. J.-P. Blaizot, Phys. Rep. 64 (1980) 171; Nucl. Phys. A649 (1999) 61c.

3. J.-P. Blaizot et al., Nucl. Phys. A591 (1995) 435.

4. G. Colò and N. Van Giai, Proceedings of the COMEX1 Conference, Paris, 2003, to appear in Nucl. Phys. A.

5. T. Duguet, Phys. Rev. C67 (2003) 044311.

6. T. Duguet and P. Bonche, Phys. Rev. C67 (2003) 054308.

7. J.W. Negele and D. Vautherin, Phys. Rev. C5 (1972) 1472; ibid. C11 10311975.

8. T.H.R. Skyrme, Phil. Mag. 11043 1956; Nucl. Phys. 96151959.

9. D. Vautherin and D.M. Brink, Phys. Rev. C3 (1972) 626.

10. J. Dechargé and D. Gogny, Phys. Rev. C21 (1980) 1568.

11. P. Gleissl et al., Ann. Phys. (N.-Y.) 197 (1990) 205.

12. B. Cochet et al., COMEX1 Conference, Paris, 2003, to appear in Nucl. Phys. A.

13. A. Akmal et al., Phys. Rev. C58 (1998) 1804. 